\title{
Fatal haemorrhage from mycotic aneurysms of the pulmonary artery
}

\author{
JM MORGAN, AD MORGAN, B ADDIS, GW BRADLEY, SG SPIRO \\ From Brompton Hospital, London, and William Harvey Hospital, Ashford, Kent
}

Septic pulmonary emboli are well known complications of intravenous drug abuse and infective endocarditis is the most common cause. Massive haemoptysis can occur from septic emboli of tricuspid valve endocarditis but is rarely a cause of death. ${ }^{1}$ Mycotic pulmonary aneurysms have, however, been recognised as a rare source of fatal haemoptysis, ${ }^{23}$ and we report a fatal haemoptysis originating in such aneurysms without evidence of endocarditis.

\section{Case report}

A 20 year old man who admitted heroin abuse was admitted with a four month history of pleuritic chest pain, weight loss, fever, and malaise. Although intravenous injection of heroin was denied, he admitted injecting, eight months before admission, an amphetamine like compound after which he developed a small suppurative lesion at the venepuncture site that spontaneously resolved. Clinical examination revealed no specific features other than low grade fever. Investigations indicated only anaemia (haemoglobin $10 \mathrm{~g} / \mathrm{dl}$ ) and polymorpholeucocytosis; blood cultures were persistently sterile. Chest radiography showed consolidation in the right lower lobe. A diagnosis of pneumonia was made and he was treated with antibiotics. The patient took his own discharge from hospital before completing medical treatment.

Eight weeks later he was readmitted with further pleuritic pain, haemoptysis, fever. A chest radiograph showed a small residual right sided effusion and a prominent left hilum (fig 1). Bacterial cultures of blood and sputum were again negative, and one week later he was referred to the Brompton Hospital for further investigation. He complained of bilateral pleuritic chest pain. Clinical examination, however, showed nothing remarkable and there were no stigmata of endocarditis. He was anaemic and had a polymorpholeucocytosis; blood cultures yielded no growth. The chest radiograph now showed a large round opacity in the left upper lobe with a left hilar shadow (fig 2). Echocardiography showed normal aortic, mitral, tricuspid, and pulmonary valves with no evidence of vegetations. At fibreoptic bronchoscopy, performed in an attempt to aspirate material for microbiological diagnosis, the left lower lobe bronchus was found to be partially occluded by extrinsic compression. After bronchial wall brushings had been taken from the left lower lobe for microbiological examination, the patient suffered an immediate haemoptysis of 2 litres. He remained haemodynamically stable but received a blood transfusion and started treatment with penicillin,

Address for reprint requests: Dr SG Spiro, Brompton Hospital, London SW3 6HP.

Accepted 30 August 1985

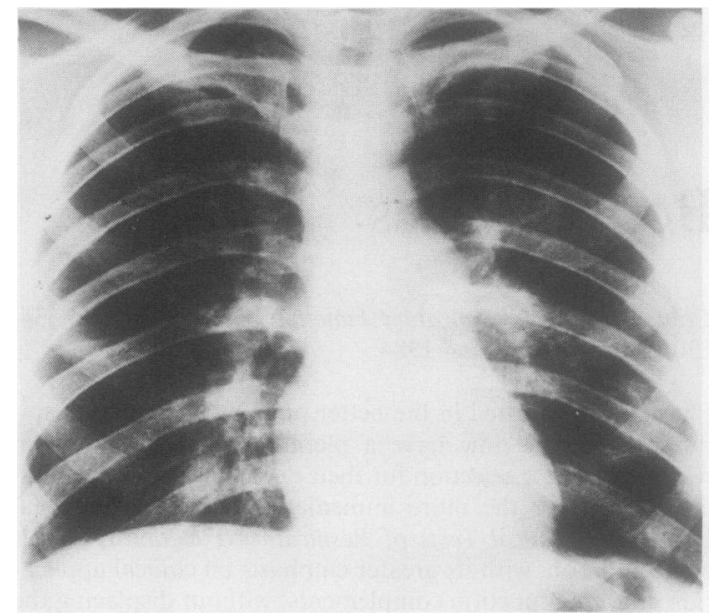

Fig 1 Chest radiograph showing a small right sided pleural effusion, a prominent left hilum, and a soft density above left hilum.

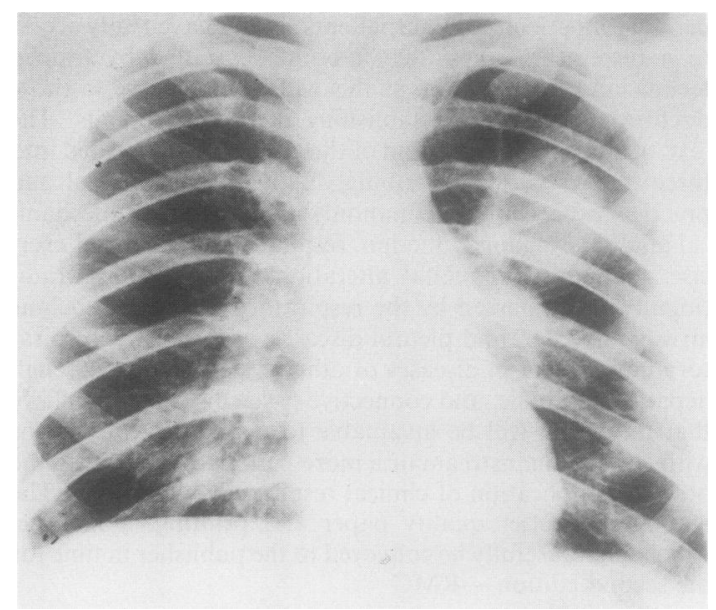

Fig 2 Chest radiograph one week later, showing a large round opacity in the left upper lobe and a prominent left hilar shadow.

flucloxacillin, and gentamicin. Cultures prepared from the lung aspirate grew Staphylococcus aureus. Fifty six hours after bronchoscopy he suffered a second haemoptysis (1.5 litres) and died. At necropsy multiple aneurysms of the pul- $\varrho$ monary arteries were present in both lungs, with the largest $\bar{\sigma}$ 
lesions in the left upper lobe. The appearances suggested that an aneurysm in the left upper lobe had ruptured, initially into the lung parenchyma and terminally into the left pleural cavity, which contained 2 litres of fresh blood. The aneurysms and other branches of the arteries contained fresh and organising thrombus. Gram stains showed only very occasional colonies of gram positive cocci and fungal stains were negative. In the heart there was a small organised vegetation adjacent to the right auricular appendage but there was no evidence of valvular damage.

\section{Discussion}

Intravenous drug abusers are at risk of endocarditis, particularly right sided endocarditis. ${ }^{3}$ Septic pulmonary emboli from vegetations may lead to cavitation and lung abscess formation, which is the commonest presentation of endocarditis of the tricuspid valve. ${ }^{45}$ Massive haemoptysis (more than $500 \mathrm{ml}$ of blood ${ }^{6}$ ) has been reported as a fatal complication of septic embolisation from tricuspid endocarditis, causing rapid suffocation. ${ }^{1}$ Mycotic aneurysms of the pulmonary tree with no obvious focal source have previously been reported as a cause of massive haemoptysis. ${ }^{23}$ In one of these cases a staphylococcal abscess of the scrotum had been present but there was subsequently no clinical evidence of endocarditis. At postmortem examination, however, a half centimetre area of opaque endocardium was found with a small, adherent, antemortem thrombus but with no valvular endocarditis. ${ }^{3}$ In our case mycotic aneurysms of the pul- monary tree presenting as a radiological opacity developed in a patient who had no clinical or microbiological evidence of endocarditis, since he had many sterile blood cultures over four months and a normal echocardiogram. The patient, who was clinically unwell, underwent bronchoscopy in an attempt to isolate a pathogen; and this procedure was likely to have contributed to his eventual death. Rapidly developing opacities on the chest radiograph without cavitation may be due to aneurysms of the pulmonary arterial tree, and pulmonary angiography should be considered before bronchoscopy to prevent a catastrophic haemorrhage.

\section{References}

1 Webb DW, Thadepalli H. Hemoptysis in patients with septic pulmonary infarcts from tricuspid endocarditis. Chest 1979;76:99-100.

2 Giraldo HD, Ramirez-Rivera J. Mycotic pulmonary artery aneurysms: a rare cause of fatal haemoptysis. Bol Asoc Med PR 1977;69:266-71.

3 Charlton RW, Du Plessis LA. Multiple pulmonary artery aneurysms. Thorax 1961;16:364.

4 Roberts WC, Buchbinder NA. Right-sided valvular infective endocarditis. A clinicopathological study of twelve necropsy patients. Am J Med 1972;53:7-19.

5 Pursel SE, Lindskog GE. Haemoptysis. A clinical evaluation of 105 patients examined consecutively on a thoracic surgical service. Am Rev Respir Dis 1961;84:329-33.

6 Committee on Therapy, American Thoracic Society. The management of hemoptysis. Am Rev Respir Dis 1966;93:472-4. 